\title{
Quantum Time Arrows, Semigroups and Time-Reversal in Scattering
}

\author{
Robert C. Bishop ${ }^{a, b}$ \\ ${ }^{a}$ Abteilung für Theorie und Datenanalyse, Institut \\ für Grenzgebiete der Psychologie, Wilhelmstrasse \\ 3a, D-79098 Freiburg, Germany \\ ${ }^{b}$ Permanent Address: Department of Philosophy, \\ Logic and Scientific Method, The London School \\ of Economics, Houghton St., London, \\ WC2A 2AE, United Kingdom \\ Accepted for publication in the International Journal of Theoretical Physics
}

\begin{abstract}
Two approaches toward the arrow of time for scattering processes have been proposed in rigged Hilbert space quantum mechanics. One, due to Arno Bohm, involves preparations and registrations in laboratory operations and results in two semigroups oriented in the forward direction of time. The other, employed by the Brussels-Austin group, is more general, involving excitations and de-excitations of systems, and apparently results in two semigroups oriented in opposite directions of time. It turns out that these two time arrows can be related to each other via Wigner's extensions of the spacetime symmetry group. Furthermore, their are subtle differences in causality as well as the possibilities for the existence and creation of time-reversed states depending on which time arrow is chosen.
\end{abstract}

Acknowledgement 1 I would like to thank I. Antoniou, H. Atmanspacher, A. Bohm, R. De La Madrid and S. Wickramasekara for illuminating discussions. Any remaining confusions are my own.

\section{Introduction}

In the standard formulation of nonrelativistic quantum mechanics, the time evolution of systems is governed by a one-parameter group of unitary operators

$$
U(t)=e^{-i H t}
$$

on a Hilbert space (HS) [18], where $H$ represents the Hamiltonian and Planck's constant has been set to one. Any evolution governed by (1) is time-reversal 
invariant $^{1}$ and irreversibility ${ }^{2}$ usually enters in due to an extrinsic act of measurement or other interaction with an environment [19,21]. This approach, however, has some undesirable features: (1) the observed exponential decay in various quantum experiments is considered as being only approximately exponential; (2) no appropriate eigenvectors describing decaying states (e.g. Gamow vectors and Dirac states) are elements of HS; (3) there is a tendency to treat metastable states such as resonances or decaying states as transients rather than as states of autonomous microphysical systems; (4) no intrinsic forms of irreversibility-where irreversible behavior originates in the dynamics of a physical system without explicit reference to an environment [1]-can be appropriately

modeled nor can appropriate initial conditions for such irreversible processes be formulated rigorously in HS.

For these, among other reasons $[3,4,8]$, theories of rigged Hilbert space (RHS) quantum mechanics-a generalization of the HS version-were developed $[2,4,5]$. A RHS, or Gel'fand triplet $[12,11]$, is the triple of spaces

$$
\Phi \subset \mathcal{H} \subset \Phi^{\times},
$$

where $\mathcal{H}$ is a HS with the standard norm topology, $\tau_{\mathcal{H}}, \Phi$ is a vector space with a topology, $\tau_{\Phi}$, stronger than $\tau_{\mathcal{H}}$ and $\Phi^{\times}$is the dual space of continuous linear functionals on $\Phi$. A RHS provides an appropriate setting for studying intrinsically irreversible processes because it naturally accommodates semigroup evolutions and the initial and boundary conditions appropriate to such evolutions [8].

In the context of scattering theory, two arrows of time intrinsic to the dynamics of quantum systems have been proposed within RHS quantum mechanics. One, due to Bohm $[7,8]$, involves preparations and registrations in laboratory operations, resulting in semigroups oriented in the forward direction of time. The other, originally proposed by George [13] and employed by the BrusselsAustin group, is more general involving excitations and de-excitations of systems, resulting in semigroups apparently oriented in opposite directions of time. I will briefly review these two quantum arrows of time and then examine their relationship under time-reversal.

\section{States and Observables}

A typical scattering experiment consists of an accelerator, which prepares a projectile in a particular state, a target and detectors. The total Hamiltonian modeling the interaction of the particle with the target is, therefore, $H=H_{o}$ $+V$, where $H_{o}$ represents the free particle Hamiltonian and $V$ the potential in the interaction region. The vectors representing growing and decaying states

\footnotetext{
${ }^{1}$ Time-reversal invariance means that if $\phi(t)$ is a solution of the quantum mechanical equations of motion, then so is $\phi(-t)$.

${ }^{2} \mathrm{~A}$ process is reversible if the temporal succession of its states $\phi_{1}, \phi_{2}, \ldots, \phi_{n}$ can occur as well as the reverse sequence of states $\phi_{n}^{T}, \phi_{n-1}^{T}, \ldots, \phi_{1}^{T}$, where $T$ is a time-reversal operation; otherwise it is irreversible.
} 
are associated with the resonance poles of the analytically continued S-matrix [14].

The preparation/registration arrow of time [8] is fundamental to Bohm's analysis of resonance states. The key intuition behind this arrow is that no observable properties of a state can be measured unless the state has first been prepared. Following Ludwig $[16,17,8]$, an in-state of a particular quantum system (considered as an ensemble of individual systems such as elementary particles) is prepared by a preparation apparatus (considered macrophysical). The detector (considered classical) registers so-called out-states of post-interaction particles. In-states are taken to be elements $\phi \in \Phi_{-}$and observables are taken to be elements $\psi \in \Phi_{+}$. (Decaying states, such as the Dirac, Lippman, Schwinger kets and Gamow vectors, are elements of $\Phi_{ \pm}^{\times}$). This leads to a distinction between prepared states and observables, each described by a separate RHS [8]:

$$
\begin{aligned}
& \Phi_{-} \subset \mathcal{H} \subset \Phi_{-}^{\times} \\
& \Phi_{+} \subset \mathcal{H} \subset \Phi_{+}^{\times},
\end{aligned}
$$

where $\Phi_{-}$is the Hardy space of the lower complex energy half-plane intersected with the Schwartz class functions and $\Phi_{+}$is the Hardy space of the upper complex energy half-plane intersected with the Schwartz class functions [8]. As Bohm and Gadella [7] demonstrate, some elements of the generalized eigenstates in $\Phi_{-}^{\times}$and $\Phi_{+}^{\times}$correspond to exponentially growing and decaying states respectively. The semigroups governing these states $\operatorname{are}^{3}$

$$
\begin{aligned}
& \left\langle\phi\left|U^{\times}\right| Z_{R}^{*}\right\rangle=e^{-i E_{R} t} e^{\frac{\Gamma}{2} t}\left\langle\phi \mid Z_{R}^{*}\right\rangle t \leq 0, t:-\infty \rightarrow 0 \\
& \left\langle\psi\left|U^{\times}\right| Z_{R}\right\rangle=e^{-i E_{R} t} e^{-\frac{\Gamma}{2} t}\left\langle\psi \mid Z_{R}\right\rangle t \geq 0, t: 0 \rightarrow \infty,
\end{aligned}
$$

where $E_{R}$ represents the total resonance energy, $\Gamma$ represents the resonance width, $Z_{R}$ represents the pole at $E_{R}-i \frac{\Gamma}{2}, Z_{R}^{*}$ represents the pole at $E_{R}+i \frac{\Gamma}{2}$, $\left|Z_{R}^{*}\right\rangle \in \Phi_{-}^{\times}$represents a growing Gamow vector and $\left|Z_{R}\right\rangle \in \Phi_{+}^{\times}$represents a decaying Gamow vector. The $t<0$ semigroup is identified as future-directed along with $\left|Z_{R}^{*}\right\rangle$ as a forming/growing state. The $t>0$ semigroup is identified as future-directed along with $\left|Z_{R}\right\rangle$ as a decaying state ${ }^{4}$.

In their discussion of scattering and resonance phenomena, Antoniou and Prigogine also apply the RHS framework, using the Hardy class functions as a natural function space for their analysis [2]. Antoniou and Prigogine adopt a time arrow somewhat different from Bohm [3]: excitations are interpreted as events taking place before $t=0$ while de-excitations are interpreted as events taking place after $t=0$. This time arrow leads to a natural splitting of the RHS:

\footnotetext{
${ }^{3}$ If $U(t)$ is a unitary operator on $\mathcal{H}$ and $\Phi \subset \mathcal{H} \subset \Phi^{\times}$, then $U^{\dagger}$ can be extended to $\Phi^{\times}$ provided that (1) $U$ leaves $\Phi$ invariant, i.e. $U: \Phi \rightarrow \Phi$, and (2) $U$ is continuous on $\Phi$ with respect to the topology $\tau_{\Phi}$. The operator $U^{\times}$denotes the extension of the HS operator $U^{\dagger}$ to $\Phi^{\times}$and is defined by $\langle U \phi \mid F\rangle=\left\langle\phi \mid U^{\times} F\right\rangle$ for all $\phi \in \Phi$ and $F \in \Phi^{\times}$. When the group operator $U^{\dagger}$ is extended to $\Phi^{\times}$, continuity requirements force the operators $U^{\times}$to be semigroups defined only on the temporal half-domains [7].

${ }^{4}$ Note that the eigenvectors plus the semigroup property are insufficient to determine the temporal direction of evolution. These identifications involve further physical justification.
} 
excitations (e.g. formation of unstable states) are considered as past-oriented and are associated with $\phi_{+} \in \Phi_{+}^{\times}$in the upper half-plane, while de-excitations (e.g. decay of ustable states) are considered as future-oriented and are associated with $\phi_{-} \in \Phi_{-}^{\times}$in the lower half-plane. ${ }^{5}$ The semigroups governing decaying states as identified by the Brussels-Austin group are

$$
\begin{aligned}
& \left\langle\phi_{+}\left|U^{\times}\right| Z_{R}^{*}\right\rangle=e^{i E_{R} t} e^{\frac{\Gamma}{2} t}\left\langle\phi_{+} \mid Z_{R}^{*}\right\rangle t<0, t:-\infty \leftarrow 0 \\
& \left\langle\phi_{-}\left|U^{\times}\right| Z_{R}\right\rangle=e^{-i E_{R} t} e^{-\frac{\Gamma}{2} t}\left\langle\phi_{-} \mid Z_{R}\right\rangle t>0, t: 0 \rightarrow \infty .
\end{aligned}
$$

The Brussels-Austin Group identifies the $t<0$ semigroup as evolving states into the past along with $\left|Z_{R}^{*}\right\rangle$ as decaying states, and the $t>0$ semigroup as evolving states into the future along with $\left|Z_{R}\right\rangle$ as decaying states.

\section{Time-reversal}

Following Wigner [20], the time-reversal operator, $R(t)$, is the HS representation of the physical spacetime transformation

$$
R:(\vec{x}, t) \rightarrow(\vec{x},-t)
$$

Therefore, $R$ is an element of a co-representation of the extended Galilei symmetry group [10] for nonrelativistic spacetime (extended Poincaré group for relativistic spacetime). These representations must be unitary and linear except for $R$, which is antilinear. With these properties, $R$ fulfils

$$
\begin{aligned}
R P_{i} R^{-1} & =-P_{i} \\
R J_{i} R^{-1} & =-J_{i} \\
R K_{i} R^{-1} & =K_{i} \\
R H R^{-1} & =H \\
R S R^{-1} & =S^{\dagger}=S^{-1},
\end{aligned}
$$

where $P_{i}, J_{i}, K_{i}, H$ and $S$ are the momentum, angular momentum, Lorentz boost, energy and S-matrix operators respectively [9]. The relation (7e) is experimentally tested in the form of the reciprocity relation, but it should be pointed out that (7) is formulated in terms of observables, not states.

However, there is one more technicality to discuss before examining the application of $R$ to the states and observables of $\S 2$. Wigner originally derived the properties of $R$ for the spacetime symmetry group extended by time inversions and studied the parity inversion operator $\Sigma$ and the total inversion operator $T$ in combination with $R$ [20]. The parity inversion operator is unitary so its phase

\footnotetext{
${ }^{5}$ Note that the roles of the upper and lower Hardy class function spaces is reversed with respect to Bohm's approach. This has only mathematical import. The differences in phase factors between (4) and (5) are due to the fact that in the former, states evolve in the Schrödinger picture while observables evolve in the Heisenberg picture, while in the latter, only the Schrödinger picture is used.
} 
can be chosen such that $\Sigma^{2}=I$ (the identity operator), while $T$ and $R$ are both anti-unitary, so that the associative law for group multiplication dictates that $R^{2}=\varepsilon_{R} I$ and $T^{2}=\varepsilon_{T} I$, where $\varepsilon_{R}= \pm 1$ and $\varepsilon_{T}= \pm 1$. The phase of $T$ can be chosen so that $T=\Sigma R$ (where the order of application of $\Sigma$ and $R$ is physically immaterial). The extension of the spacetime symmetry group is summarized in Table 1.

\begin{tabular}{|c|c|c|c|c|}
\hline$\varepsilon_{R}$ & $\varepsilon_{T}$ & $\Sigma$ & $R$ & $T$ \\
\hline$(-1)^{2 j}$ & $(-1)^{2 j}$ & 1 & $C$ & $C$ \\
\hline$-(-1)^{2 j}$ & $(-1)^{2 j}$ & $\left(\begin{array}{cc}1 & 0 \\
0 & -1\end{array}\right)$ & $\left(\begin{array}{cc}0 & C \\
-C & 0\end{array}\right)$ & $\left(\begin{array}{cc}0 & C \\
C & 0\end{array}\right)$ \\
\hline$(-1)^{2 j}$ & $-(-1)^{2 j}$ & $\left(\begin{array}{cc}1 & 0 \\
0 & -1\end{array}\right)$ & $\left(\begin{array}{cc}0 & C \\
C & 0\end{array}\right)$ & $\left(\begin{array}{cc}0 & C \\
-C & 0\end{array}\right)$ \\
\hline$-(-1)^{2 j}$ & $-(-1)^{2 j}$ & $\left(\begin{array}{cc}1 & 0 \\
0 & 1\end{array}\right)$ & $\left(\begin{array}{cc}0 & C \\
-C & 0\end{array}\right)$ & $\left(\begin{array}{cc}0 & C \\
-C & 0\end{array}\right)$ \\
\hline
\end{tabular}

The index $j$ refers to the spin of the particle being considered while $C$ is an operator whose $(2 j+1)$-dimensional matrix has the elements $c_{\mu, \nu}=(-1)^{j+\mu} \delta_{\mu, \nu}$, where $-j \leq \mu$ and $\nu \leq j$. In the first representation, where $\varepsilon_{R}=\varepsilon_{T}=(-1)^{2 j}$, there are no changes to the underlying vector space. This is the typical case discussed in quantum mechanics (and relativistic quantum field theory). The other three representations, however, exhibit a doubling of the vector spaces. In order to track this space doubling, let the index $r=0,1$ label the rows and columns of the operator matrices in Table 1.

\section{Time-reversed States and Observables}

Although no quantum fields have been constructed for representations two and three of Table 1 (indeed they are highly problematic), Bohm and co-workers have constructed models for the fourth representation by applying $\mathrm{R}$ to the states and observables in (4) $[6,9]$. First, consider the growing Gamow vectors for, $\phi^{r=0, \times} \in \Phi_{-}^{r=0, \times}$. Applying $R$ yields

$$
R \phi^{r=0, \times}=\psi^{r=1, \times} \in \Phi_{+}^{r=1, \times} .
$$

Similarly for the decaying Gamow vectors, $\psi^{r=0, \times} \in \Phi_{+}^{r=0, \times}$, applying $R$ yields

$$
R \psi^{r=0, \times}=\phi^{r=1, \times} \in \Phi_{-}^{r=1, \times} .
$$

The transformation properties of $R$ may be summarized as $R: \Phi_{ \pm}^{r=0, \times} \rightarrow$ $\Phi_{\mp}^{r=1, \times}$. The temporal evolution of these time-reversed vectors is also given by semigroups. Identify $r=0$ with the scattering experiment as normally carried out in the laboratory and $r=1$ with the time-reversed situation. Then $U^{\times}(t)\left\langle\phi, r=0 \mid Z_{R}^{*}, r=0\right\rangle \in \Phi_{-}^{r=0, \times}$, a growing Gamow vector representing a preparable state for $t \leq 0$, is transformed under $R$ into $U^{\times}(-t)\langle\psi, r=1| Z_{R}, r=$ $1\rangle \in \Phi_{+}^{r=1, \times}$, where

$$
e^{i E_{R} t} e^{-\frac{\Gamma}{2} t}\left\langle\psi, r=1 \mid Z_{R}, r=1\right\rangle
$$


is restricted to the time domain $t \geq 0$ by continuity requirements. In the case of $\left|Z_{R}^{*}, r=0\right\rangle$, time runs from $-\infty$ to 0 ; in contrast, for $\left|Z_{R}, r=1\right\rangle$, time runs from $\infty$ to 0 , meaning that it represents a Gamow vector that increases as $t$ decreases. Similarly, $U^{\times}(t)\left\langle\psi, r=0 \mid Z_{R}, r=0\right\rangle \in \Phi_{+}^{r=0, \times}$, a decaying Gamow vector representing observables for $t \geq 0$, is transformed under $R$ into $U^{\times}(-t)\left\langle\phi, r=1 \mid Z_{R}^{*}, r=1\right\rangle \in \Phi_{-}^{r=1, \times}$, where

$$
e^{i E_{R} t} e^{\frac{\Gamma}{2} t}\left\langle\phi, r=1 \mid Z_{R}^{*}, r=1\right\rangle
$$

is restricted to the time domain $t \leq 0$ by continuity requirements. In the case of $\left|Z_{R}, r=0\right\rangle$, time runs from 0 to $\infty$; in contrast, for $\left|Z_{R}^{*}, r=1\right\rangle$, time runs from 0 to $-\infty$, meaning that it represents a Gamow vector that decays as $-t$ increases. These results are summarized in Table 2.

\begin{tabular}{|l|l|l|}
\hline Growing & $\left\langle\phi, r=0 \mid Z_{R}^{*}, r=0\right\rangle$ & $\left\langle\psi, r=1 \mid Z_{R}, r=1\right\rangle$ \\
\hline Vectors & $t \leq 0, t:-\infty \rightarrow 0$ & $t \geq 0, t: 0 \leftarrow \infty$ \\
\hline & & \\
\hline Decaying & $\left\langle\psi, r=0 \mid Z_{R}, r=0\right\rangle$ & $\left\langle\phi, r=1 \mid Z_{R}^{*}, r=1\right\rangle$ \\
\hline Vectors & $t \geq 0, t: 0 \rightarrow \infty$ & $t \leq 0, t:-\infty \leftarrow 0$ \\
\hline
\end{tabular}

The time-reversed situation in the Brussels-Austin approach have not been discussed in the literature. Using the transformation rules as appropriate, the temporal evolution of the time-reversed vectors can be determined. However, notice that the eigenvectors in (5) are identified with decaying states. It can be easily seen that (5b) is the time-reversal of $(5 \mathrm{a})$ under $R$, but the label $r$ associated with vector space doubling remains to be identified. If we assume that the preparation/registration arrow is a special case of the excitation/deexcitation arrow-that is, that laboratory preparations are particular types of excitations and the detections of decaying states are particular types of deexcitations [3]-then (5a) can be identified with the $r=1$ and (5b) with the $r=0$ regimes respectively (compare with $(4 \mathrm{~b})$ ).

What remains is to examine the eigenvectors representing growing states in the Brussels-Austin approach. To each de-excitation in (5) there is a corresponding excitation represented by an eigenvector in the opposite temporal half-plane. For the $r=0$ regime, a growing eigenvector of the form

$$
e^{i E_{R} t} e^{\frac{\Gamma}{2} t}\left\langle\phi_{+}, r=0 \mid Z_{R}^{*}, r=0\right\rangle,
$$

corresponds to eigenstate $(5 \mathrm{~b})$, where (12) is restricted to the time domain $t<0$ by continuity requirements. This state is represented by a Gamow vector that grows as $-t$ decreases. Similarly, for the $r=1$ regime, a growing eigenstate of the form

$$
e^{-i E_{R} t} e^{-\frac{\Gamma}{2} t}\left\langle\phi_{-}, r=1 \mid Z_{R}, r=1\right\rangle,
$$

corresponds to eigenvector (5a), where (13) is restricted to the time domain $t>0$ by continuity requirements. This state is represented by a Gamow vector that grows as $t$ decreases. These results are summarized in Table 3 . 


\begin{tabular}{|l|l|l|}
\hline Growing & $\left\langle\phi_{+}, r=0 \mid Z_{R}^{*}, r=0\right\rangle$ & $\left\langle\phi_{-}, r=1 \mid Z_{R}, r=1\right\rangle$ \\
\hline Vectors & $t<0, t:-\infty \rightarrow 0$ & $t>0, t: 0 \leftarrow \infty$ \\
\hline & & \\
\hline Decaying & $\left\langle\phi_{-}, r=0 \mid Z_{R}, r=0\right\rangle$ & $\left\langle\phi_{+}, r=1 \mid Z_{R}^{*}, r=1\right\rangle$ \\
\hline Vectors & $t>0, t: 0 \rightarrow \infty$ & $t<0, t:-\infty \leftarrow 0$ \\
\hline
\end{tabular}

The Bohm and Brussels-Austin groups appear to be working with the same eigenvectors and semigroups in their analyses of scattering. (5a) and (5b) are time-reversed images of each other and, when paired with their corresponding growing vectors, are easily related to those of Bohm and co-workers (compare Tables 2 and 3), which is not immediately apparent when comparing (4) and (5) without taking time reversal and vector space doubling into account.

\section{The Possibility of Time-reversed States}

It has been suggested that (5a) be disregarded because it is inconsistent with observations or because of other consistency requirements such as the need for devices to communicate $[2,3]$. Does the consideration of time-reversed states in the light of vector space doubling lead to new arguments for disregarding (5a)?

\subsection{Physical Considerations}

Lee [15] discusses the following problem with time-reversed quantum states. Consider a $\bar{\mu}$-meson at rest with its spin $\mathbf{s}_{\mu}$ in the up direction. It decays as

$$
\bar{\mu} \rightarrow e^{-}(L)+\bar{\nu}_{e}(R)+\nu_{\mu}(L),
$$

where the electron, electron anti-neutrino and $\mu$ neutrino are emitted with helicities $-1 / 2,1 / 2$ and $-1 / 2$ respectively, denoted by the letters $L$ and $R$ indicating the helicities. Neglecting the electronic mass and assuming that the final momenta of $e^{-}, \bar{\nu}_{e}$ and $\nu_{\mu}$ are $\mathbf{P}_{e}, \mathbf{P}_{\bar{\nu}}$ and $\mathbf{P}_{\nu}$, respectively, the time-reversed process would be

$$
e^{-}(L)+\bar{\nu}_{e}(R)+\nu_{\mu}(L) \rightarrow \bar{\mu},
$$

where the initial states of $e^{-}, \bar{\nu}_{e}$ and $\nu_{\mu}$ have momenta $-\mathbf{P}_{e},-\mathbf{P}_{\bar{\nu}}$ and $-\mathbf{P}_{\nu}$ respectively. If time reversal holds, then (15) should lead to a final state with $\bar{\mu}$ at rest. Also (15) should produce a final spin $\mathbf{s}_{\mu}^{\prime}=-\mathbf{s}_{\mu}$, but this is not generally the case in quantum mechanics. For example, if the momenta of $\bar{\nu}_{e}$ and $\nu_{\mu}$ are parallel in (14), then conservation of total angular momentum in (15) requires that $\mathbf{s}_{\mu}^{\prime}$ lie in the same direction as the initial electron spin, which is typically different from that of $-\mathbf{s}_{\mu}$. In the more general case, where the directions of the momenta in (14) are arbitrary, the final spin $\mathbf{s}_{\mu}^{\prime}=-\mathbf{s}_{\mu}$ in (15) is only possible if the momentum and spin of all three leptons are simultaneously reversed in all possible directions while maintaining the appropriate phase relations among their wave amplitudes. The latter would require the creation of three perfectly 
coherent incoming spherical waves in the midst of the many degrees of freedom involved.

Producing such a state in laboratory situations (preparation/registration arrow) is clearly impossible because the precision required to produce such coherent incoming spherical waves, as well as the control over the environment it entails, exceeds our engineering capabilities (presuming we knew how to produce such phase-related time-reversed waves). For more general unstable quantum processes (excitation/de-excitation arrow), it is not clear that time-reversed growing states associated with the $r=1$ regime can be ruled out so easily. Though highly improbable, perhaps some kinds of singular events can produce the kinds of time-reversed processes meeting such stringent requirements.

There is a related question as to why we live in a universe where the overwhelming proportion of processes are in the $r=0$ regime [6]. This would be the case if the initial explosion of the big bang singularity was a process of type $r=0$. All subsequent processes would then typically be of type $r=0$ with the possible exception of exceedingly rare, highly singular processes producing a type $r=1$ event. However, the sheer preponderance of $r=0$ processesincluding the "master $r=0$ process," the cosmic arrow-implies an improbably high entropy barrier that such rare $r=1$ processes must overcome.

\subsection{Causal Considerations}

One might also argue against time-reversed processes by invoking a standard formulation of the causal relation between events: causes must precede their effects in temporal order. However, the more general form of the causal relation is that causes must precede their effects in logical order, leaving open the possibility for backwards-in-time causation. For the preparation-registration arrow, such causal considerations present problems for $r=1$ type processes. The preparation of states $\phi$ is required before observables $\psi$ can be measured because observables logically presuppose states $[4,8]$. The $r=1$ regime appears to contradict this causality requirement in that observables $\psi$ are "prepared" before states $\phi$ can be "measured." This is to say, that $R$ interchanges the roles of states and observables. If observables are logically dependent on states, then one might argue that there must be some kind of (strange) state in the $r=1$ regime for $t>0$ unaccounted in Table 2, but the production of such states presents insurmountable difficulties ( $\$ 5.1)$.

For the more general case of the excitation/de-excitation arrow, causal considerations do not necessarily rule out time-reversed states. For the $r=0$ regime, excitations $\phi_{+}$lead to de-excitations $\phi_{-}$(e.g. by emitting some decay product leading to de-excitation). In contrast for the $r=1$ regime, the transformation rules indicate that de-excitations $\phi_{-}$lead to excitations $\phi_{+}$, as again the roles of the vector spaces become interchanged. That suggests the identification of (5a) as a de-excitation into the past is not unique. If we keep this latter identification of decay into the past, there is nothing more to be said, as there is neither a temporal nor a logical relationship specifying the order of excitation and de-excitation. There are only the improbability considerations 
described above.

Even if we modify the identification as the $r=1$ regime suggests, this does not immediately lead to an argument ruling out $r=1$ processes because again the logical form of the causal relation does not foreclose the possibility that deexcited states may become re-excited in a time reversed fashion. There are two cases. First, the spontaneous excitation could be self-caused, but this violates the causal relation in that all effects must have a cause. The only possibility in this case is an uncaused event, sheer chance. Second, some process leads to the spontaneous excitation of the de-excited state into the past. The Unruh effect, where some kinds of ground states can be spontaneously excited even when moving through vacuum, and pair production are possible mechanisms in quantum field theory, but these effects are not immediately applicable if we restrict ourselves to standard quantum mechanics.

\section{3 "Weirdness" Considerations}

However, it does appear that the $r=1$ regime presents an interpretive difficulty. Under the registration-preparation arrow, observables are now represented by growing eigenvectors while states are represented by decaying eigenvectors. Under the excitation/de-excitation arrow, if one follows what the transformation rules suggest, de-excitations are represented by growing eigenvectors while excitations are represented by decaying eigenvectors. These associations are clearly not as natural as those in the $r=0$ regime, perhaps suggesting some as yet undiscovered problems with the fourth representation of Table 1.

\section{Discussion}

It appears that the time-reversal invariance of the dynamics in conventional quantum mechanics is due to the underlying symmetries of the HS in which it is formulated. This time-reversal symmetry is missing from the RHS generalization for the case of resonance phenomena. Nevertheless, it may be possible to restore some form of time-reversal symmetry in RHS quantum mechanics via the extended spacetime symmetry group. For the registration/preparation arrow, while the formalism allows states (and observables) to be distinguished from their time-reversed counterparts, such counterparts are not physically possible. For the more general excitation/de-excitation arrow, time-reversed counterparts may also be distinguished, but appear to be only highly improbable.

However, there is something weird about the fourth extended spacetime representation and the rareness of $r=1$ processes may be related to this weirdness. If there turns out to be a serious problem with this representation (e.g. a problematic unexamined assumption) such that it must be discarded, then time-reversed states would disappear from RHS quantum mechanics in the context of resonance phenomena as unphysical, leaving a purely time-asymmetric theory. 


\section{References}

1. H. Atmanspacher and R. Bishop, A. Amann, Extrinsic and Intrinsic Irreversibility in Probabilistic Dynamical Laws, in A. Khrennikov (ed.) Quantum Probability and White Noise Analysis Volume XIII, World Scientific, Singapore (2002), 50-70.

2. I. Antoniou and I. Prigogine, Physica A 192, (1993) 443-64.

3. R. Bishop, Int. J. Theor. Phys. (forthcoming).

4. A. Bohm, Rigged Hilbert Space and Mathematical Description of Physical Systems, in W. Brittin, et al. (eds.) Lectures in Theoretical Physics Vol IX A: Mathematical Methods of Theoretical Physics, Gordon and Breach Science Publishers, New York (1967) 255-317.

5. A. Bohm, The Rigged Hilbert Space and Quantum Mechanics, Springer, Berlin (1978).

6. A. Bohm, Physical Review A 51, (1995) 1758-69.

7. A. Bohm and M. Gadella, Dirac Kets, Gamow Vectors, and Gel'fand Triplets, Lecture Notes in Physics, vol. 348, Springer, Berlin (1989).

8. A. Bohm, S. Maxson, M. Loewe and M. Gadella, Physica A 236, (1997) 485-549.

9. A. Bohm and S. Wickramasekara, Foundations of Physics 27, (1997) 969-93.

10. J. Cariñena and M. Santander, J. Math. Phys. 22, (1981) 1548.

11. I. Gel'fand and G. Shilov, Generalized Functions Volume 3: Theory of Differential Equations, M. Mayer (tr.), Academic Press, New York (1967).

12. I. Gel'fand and N. Vilenkin, Generalized Functions Volume 4: Applications of Harmonic Analysis, A. Feinstein (tr.), Academic Press, New York (1964).

13. C. George, Bulletin de la Classe des Sciences Academie Royale des Sciences, des Lettres et des Beaux-Arts de Belgique 56, (1971), 505

14. P. Lax and R. Phillips, Scattering Theory, Academic Press, New York (1967).

15. T. Lee, Particle Physics and Introduction to Field Theory, Harwood Academic Publishers, New York (1981).

16. G. Ludwig, Foundations of Quantum Mechanics, Vol. I, Springer, Berlin (1983).

17. G. Ludwig, Foundations of Quantum Mechanics, Vol. II, Springer, Berlin (1985).

18. J. von Neumann, Mathematical Foundations of Quantum Mechanics, Princeton University Press, Princeton (1955/1932).

19. J. Wheeler and W. Zurek, Quantum Theory and Measurement, Princeton University Press, Princeton (1988).

20. E. Wigner, Unitary Representations of the Inhomogeneous Lorentz Group Including Reflections, in Group Theoretical Concepts and Methods in Elementary Particle Physics, F. Gürsey (ed.), Gordon and Breach, Science Publishers, New York: (1964). 
21. H. Zeh, The Physical Basis of the Direction of Time, Third Edition, Springer, Berlin (1999). 